University of Nebraska - Lincoln

DigitalCommons@University of Nebraska - Lincoln

$1-27-2015$

\title{
The Body Size Dependence of Trophic Cascades
}

John DeLong

Benjamin Gilbert

Jonathan B. Shurin

Van M. Savage

Brandon T. Barton

See next page for additional authors

Follow this and additional works at: https://digitalcommons.unl.edu/bioscifacpub

Part of the Biology Commons

This Article is brought to you for free and open access by the Papers in the Biological Sciences at DigitalCommons@University of Nebraska - Lincoln. It has been accepted for inclusion in Faculty Publications in the Biological Sciences by an authorized administrator of DigitalCommons@University of Nebraska - Lincoln. 


\section{Authors}

John DeLong, Benjamin Gilbert, Jonathan B. Shurin, Van M. Savage, Brandon T. Barton, Christopher F. Clements, Anthony I. Dell, Hamish S. Greig, Christopher D.G. Harley, Pavel Kratina, Kevin S. McCann, Tyler D. Tunney, David A. Vasseur, and Mary I. O'Connor 


\title{
The Body Size Dependence of Trophic Cascades
}

\author{
John P. DeLong, ${ }^{1, \star}$ Benjamin Gilbert, ${ }^{2}$ Jonathan B. Shurin, ${ }^{3}$ Van M. Savage, ${ }^{4}$ Brandon T. Barton, ${ }^{5}$ \\ Christopher F. Clements, ${ }^{6}$ Anthony I. Dell, ${ }^{7}$ Hamish S. Greig, ${ }^{8}$ Christopher D. G. Harley, ${ }^{9}$ \\ Pavel Kratina, ${ }^{10}$ Kevin S. McCann, ${ }^{11}$ Tyler D. Tunney, ${ }^{11}$ David A. Vasseur, ${ }^{12}$ and \\ Mary I. O'Connor'
}

1. School of Biological Sciences, University of Nebraska, Lincoln, Nebraska 68588; 2. Department of Ecology and Evolutionary Biology, University of Toronto, Toronto, Ontario M5S 3G5, Canada; 3. University of California, San Diego, California 92093; 4. Department of Biomathematics, David Geffen School of Medicine at UCLA, Los Angeles, California 90095; and Santa Fe Institute, Santa Fe, New Mexico 87501; 5. Department of Zoology, University of Wisconsin, Madison, Wisconsin 53589; 6. Institute of Evolutionary Biology and Environmental Studies, University of Zurich, Zurich CH-8057, Switzerland; 7. Systemic Conservation Biology, Department of Biology, University of Gottingen, Gottingen 37073, Germany; and National Great Rivers Research and Education Center, East Alton, Illinois 62024; 8. School of Biology and Ecology, University of Maine, Orono, Maine 04469; 9. Department of Zoology and Biodiversity Research Centre, University of British Columbia, Vancouver, British Columbia V6T 1Z4, Canada; 10. School of Biological and Chemical Sciences, Queen Mary University of London, London E14NS, United Kingdom; 11. Department of Integrative Biology, University of Guelph, Guelph, Ontario N1G 2W1, Canada; 12. Department of Ecology and Evolutionary Biology, Yale University, New Haven, Connecticut 06511

Submitted July 1, 2014; Accepted November 12, 2014; Electronically published January 27, 2015

Online enhancement: appendix. Dryad data: http://dx.doi.org/10.5061/dryad.74854.

ABSTRACT: Trophic cascades are indirect positive effects of predators on resources via control of intermediate consumers. Larger-bodied predators appear to induce stronger trophic cascades (a greater rebound of resource density toward carrying capacity), but how this happens is unknown because we lack a clear depiction of how the strength of trophic cascades is determined. Using consumer resource models, we first show that the strength of a trophic cascade has an upper limit set by the interaction strength between the basal trophic group and its consumer and that this limit is approached as the interaction strength between the consumer and its predator increases. We then express the strength of a trophic cascade explicitly in terms of predator body size and use two independent parameter sets to calculate how the strength of a trophic cascade depends on predator size. Both parameter sets predict a positive effect of predator size on the strength of a trophic cascade, driven mostly by the body size dependence of the interaction strength between the first two trophic levels. Our results support previous empirical findings and suggest that the loss of larger predators will have greater consequences on trophic control and biomass structure in food webs than the loss of smaller predators.

Keywords: trophic cascade, allometry, body size scaling, interaction strength, predator-prey size ratio, food chain.

\section{Introduction}

Trophic cascades, or indirect positive effects of predators on primary producers, are a dominant paradigm in our

* Corresponding author; e-mail: jpdelong@unl.edu.

Am. Nat. 2015. Vol. 185, pp. 354-366. (C) 2015 by The University of Chicago. 0003-0147/2015/18503-55590\$15.00. All rights reserved.

DOI: $10.1086 / 679735$ understanding of ecosystem structure and function (Hairston et al. 1960; Hrbacek et al. 1961; Paine 1980; Carpenter et al. 1985; Terborgh and Estes 2010). Through trophic cascades, basal resource production can be regulated not only by nutrient limitation but also by top-down control. By linking diversity and abundance across trophic levels, trophic cascades play an important role in mediating changes in the structure and function of ecological communities. For example, trophic cascades can determine the ecosystem-scale consequences of predator extinction (Estes et al. 2011), influence biogeochemical cycles and greenhouse gas dynamics (Wilmers et al. 2012; Atwood et al. 2013; Strickland et al. 2013), and naturally reduce pest abundance and increase agricultural yields (Costamagna et al. 2007).

Although predators often influence the structure and function of the ecosystems they inhabit (Estes et al. 2011), a number of studies have failed to observe a change in primary productivity or plant biomass following predator removal in both terrestrial (Meserve et al. 2003; Maron and Pearson 2011) and aquatic (Elser et al. 1998) systems. Furthermore, the strength of a trophic cascade can vary with environmental conditions and species composition and diversity (Polis 1999; Chase 2000; Frank et al. 2006; Shurin et al. 2006; Barton et al. 2009; Kratina et al. 2012; Tunney et al. 2012; Burkepile 2013). Such variation in the strength or occurrence of trophic cascades fosters debate as to their ubiquity (Laws and Joern 2013). Identifying the drivers of this variation may explain and help anticipate 
when the loss of top predators will have ecological consequences. This knowledge is of immediate importance because predators are particularly imperiled in the contemporary world (Ripple et al. 2014).

Body size is a major driver of the strength of consumerresource interactions due to the influence of size on energy requirements and the way body size influences gape size, detection limits, and other aspects of prey detection and selection (Yodzis and Innes 1992; Emmerson et al. 2006; Vucic-Pestic et al. 2010; DeLong and Vasseur 2012a; Pawar et al. 2012). Considering this relationship between body size and interaction strengths in consumer-resource interactions, it seems reasonable to expect that body size relationships may play a vital role in setting the strength of top-down control. Current evidence suggests that larger predators appear to impose stronger top-down control, allowing greater release of autotrophs from herbivory (Borer et al. 2005; Jochum et al. 2012; Simonis 2013), but the mechanisms behind this pattern are not well understood. One allometric food chain model predicts that the strength of trophic cascades should not be influenced by predator size alone but rather by predator-prey size ratios (Shurin and Seabloom 2005). Yet the effect of predatorprey size ratios on interaction strengths appears inconsistent, sometimes showing a positive correlation or no correlation with body size ratio (Emmerson and Raffaelli 2004; Emmerson et al. 2006) and other times peaking at intermediate ratios (Vucic-Pestic et al. 2010). It is also possible that the observed effect of predator size or size ratio on the strength of trophic cascades is confounded with habitat type if predator body size varies among habitats. Thus, a mechanistic theoretical framework is needed to determine how predator body size per se influences the strength of predator-prey interactions and, therefore, the strength of a potential trophic cascade, independent of other factors. This framework must begin with a general understanding of what sets the strength of a trophic cascade.

In this study, we use consumer-resource models to explore the strength of trophic cascades. We derive a new expression for the strength of a trophic cascade and use it to address two primary questions. First, how do interaction strengths (defined as the ratio of a species' abundance without a consumer to the abundance with a consumer) influence the strength of a trophic cascade? Second, how does predator body size alter the strength of trophic cascades? Our results suggest that the source of the body size dependence of trophic cascades is the body size dependence of lower trophic interactions. Our model paves the way for explicit tests of our theory in predicting the strength of a trophic cascade across body size, temperature, and other gradients.

\section{Methods}

\section{Deriving a Dynamic Model for a Trophic Cascade}

Consider a three-trophic-level food chain where a basal resource ( $R$; typically an autotroph, although in many aquatic and soil systems it could be a heterotroph) is consumed by a consumer $(C)$, and the consumer is eaten by a predator $(P)$. Following Borer et al. (2005), we define a trophic cascade (TC) as the ratio of the equilibrium abundance of the basal resource when occurring with its consumer and a predator $\left(\hat{R}_{3}\right)$ to the equilibrium abundance of a resource when occurring only with its consumer $\left(\hat{R}_{2}\right)$ :

$$
\mathrm{TC}=\frac{\hat{R}_{3}}{\hat{R}_{2}} .
$$

Shurin and Seabloom (2005) used a similar but inverse definition of a trophic cascade. A higher value of TC indicates a stronger indirect effect of predators on basal resource abundance. The subscripts define the length of the trophic chain at which the equilibrium is calculated. We then define a three-trophic-level food chain using a LotkaVolterra model with logistic growth in the resource:

$$
\begin{aligned}
\frac{\mathrm{d} R}{\mathrm{~d} t} & =r R\left(1-\frac{R}{K}\right)-a_{\mathrm{c}} R C, \\
\frac{\mathrm{d} C}{\mathrm{~d} t} & =e_{\mathrm{c}} a_{\mathrm{c}} R C-a_{\mathrm{p}} C P-m_{\mathrm{c}} C, \\
\frac{\mathrm{d} P}{\mathrm{~d} t} & =e_{\mathrm{p}} a_{\mathrm{p}} C P-m_{\mathrm{p}} P,
\end{aligned}
$$

where $r$ is the growth rate of the basal resource species and $K$ is its carrying capacity. The consumers (parameters with a subscript $c$ ) and predators (parameters with a subscript p) clear space of their prey at rate $a$ (i.e., area of capture or attack efficiency), convert prey into new consumers with efficiency $e$, and die at intrinsic mortality rate $m$ (all parameters and units are described in table 1). In the main text, we develop models with type 1 functional responses because they are mathematically more tractable, and in the appendix (available online) we develop and parameterize an analogous model with a type 2 functional response to explore when these different functional responses alter model predictions. The type 1 functional response provides accurate depictions of the body size scaling of abundance (DeLong and Vasseur 2012a, 2012b; appendix), and we therefore restrict our main discussion to equations (2)-(4). This is a simplified community module and is not intended to fully represent complex food webs (Polis and Strong 1996).

The equilibrium resource abundance can be obtained 
Table 1: Parameters used in the models and their allometric relationships

\begin{tabular}{|c|c|c|c|c|c|}
\hline \multicolumn{3}{|c|}{ Scaling functions } & \multicolumn{3}{|c|}{ Empirical estimates for protist and algae scaling functions } \\
\hline Parameter & Description & $\begin{array}{l}\text { Body size } \\
\text { scaling }\end{array}$ & Unit & Intercept & Exponent \\
\hline$r$ & $\begin{array}{l}\text { Maximum population } \\
\text { growth rate of } \\
\text { resource }\end{array}$ & $r_{0} M_{r}^{\rho}$ & day $^{-1}$ & $5.25(3.97,7.54)$ & $-.20(-.25,-.16)$ \\
\hline K & $\begin{array}{l}\text { Carrying capacity of } \\
\text { resource }\end{array}$ & $k_{0} M_{r}^{\kappa}$ & cells $\mathrm{mL}^{-1}$ & $5.83 \mathrm{E}+08(3.84 \mathrm{E}+08,1.06 \mathrm{E}+09)$ & $-.81(-.88,-.74)$ \\
\hline$a$ & $\begin{array}{l}\text { Attack efficiency of } \\
\text { consumer/predator }\end{array}$ & $a_{0} M_{c, p}^{\alpha}$ & $\mathrm{mL}$ pred $^{-1}$ day $^{-1}$ & $1.20 \mathrm{E}-06(2.19 \mathrm{E}-07,6.39 \mathrm{E}-06)$ & $1.00(.86,1.16)$ \\
\hline$e$ & $\begin{array}{l}\text { Conversion efficiency of } \\
\text { consumer/predator }\end{array}$ & $e_{0} M_{c, p}^{\varepsilon}$ & pred $_{\text {prey }}{ }^{-1}$ & $2.16(.87,5.71)$ & $-.50(-.60,-.41)$ \\
\hline$m$ & $\begin{array}{l}\text { Mortality rate of con- } \\
\text { sumer/predator }\end{array}$ & $m_{0} M_{c, p}^{\mu}$ & day $^{-1}$ & $5.62(2.75,13.38)$ & $-.29(-.35,-.22)$ \\
\hline$M_{\mathrm{r}}, M_{\mathrm{c}}$ & $\begin{array}{l}\text { Size of species being } \\
\text { consumed (basal re- } \\
\text { source or consumer) }\end{array}$ & $s_{0} M_{c, p}^{\psi}$ & $\mu \mathrm{m}^{3}$ & $2.16(.46,7.62)$ & $.66(.52, .82)$ \\
\hline
\end{tabular}

Note: For simplicity, intercepts in the allometric relationships are given as lowercase Latin letters subscripted with 0 , and all exponents are given by their closest Greek counterparts. Fitted parameters ( $\pm 95 \%$ bootstrapped confidence intervals) are shown for intercepts and exponents averaged from ordinary least squares and reduced major axis regressions.

from equation (2) and is $\hat{R}_{3}=K\left(1-a_{\mathrm{c}} \hat{C}_{3} / r\right)$, where $\hat{C}_{3}$ is the equilibrium consumer density in the three-trophiclevel model. Substituting this into equation (1) gives

$$
\mathrm{TC}=\frac{K}{\hat{R}_{2}}\left(1-\frac{a_{\mathrm{c}} \hat{C}_{3}}{r}\right) .
$$

From equation (2), we find that the equilibrium consumer density in the absence of the predator is

$$
\hat{C}_{2}=\frac{r}{a_{c}}\left(1-\frac{\hat{R}_{2}}{K}\right),
$$

and so

$$
\frac{a_{\mathrm{c}}}{r}=\frac{1-\left(\hat{R}_{2} / K\right)}{\hat{C}_{2}}
$$

which we substitute into equation (5) to yield

$$
\mathrm{TC}=\frac{K}{\hat{R}_{2}}\left(1-\frac{\left[1-\left(\hat{R}_{2} / K\right)\right] \hat{C}_{3}}{\hat{C}_{2}}\right) .
$$

Equation (6) shows how a trophic cascade is composed of interacting effects of the equilibrium abundance of the consumers and the resource species. This same structure arises when a saturating type 2 functional response is used (appendix).

\section{Modeling a Trophic Cascade in Terms of Constituent Interaction Strengths}

Equation (6) gives an explicit definition of a trophic cascade in terms of the equilibrium abundances of the consumer and basal resources. The ratio of equilibrium abundances of a particular species with and without its consumer reflects underlying dynamics of per capita resource use rates that drive the strength of the overall indirect interaction. This abundance ratio is a common definition of interaction strength among trophically linked populations (Berlow et al. 2004; Wootton and Emmerson 2005), and we can use this to simplify the model and increase our mechanistic understanding of trophic cascades. We define the interaction strength between the first and second trophic levels as IS ${ }_{12}=\hat{R}_{1} / \hat{R}_{2}=K / \hat{R}_{2}$ and the interaction strength between the second and third trophic levels as $\mathrm{IS}_{23}=\hat{C}_{2} / \hat{C}_{3}$. Substituting into equation (6) and simplifying yields an expression for the strength of a trophic cascade that is equivalent to equation (6) but expressed in terms of interaction strengths (IS):

$$
\begin{aligned}
\mathrm{TC}_{\mathrm{IS}} & =\mathrm{IS}_{12}\left(1-\frac{\left[1-\left(1 / \mathrm{IS}_{12}\right)\right]}{\mathrm{IS}_{23}}\right) \\
& =\mathrm{IS}_{12}-\frac{\left(\mathrm{IS}_{12}-1\right)}{\mathrm{IS}_{23}} .
\end{aligned}
$$

The top-down effect of predators can cascade across more than three trophic levels in natural ecosystems (Estes et al. 1998). To extend our approach to these longer food chains, we assessed how defining a trophic cascade as 
Table 2: Model components, parameter-based equilibria, and dependence on the body size of the predator

\begin{tabular}{|c|c|c|}
\hline Model component & Model expression & $\begin{array}{l}\text { Dependence on body size } \\
\text { of top predator }\end{array}$ \\
\hline $\begin{array}{l}\text { Equilibrium abundance of resource in two-trophic-level } \\
\text { food chain }\end{array}$ & $\hat{R}_{2}=\frac{m_{\mathrm{c}}}{e_{\mathrm{c}} a_{\mathrm{c}}}$ & $\hat{R}_{2}=\frac{m_{0} s_{0}^{\mu-\varepsilon-\alpha}}{e_{0} a_{0}} M_{\mathrm{p}}^{\psi(\mu-\varepsilon-\alpha)}$ \\
\hline $\begin{array}{l}\text { Equilibrium abundance of resource in one-trophic-level } \\
\text { food chain }\end{array}$ & K & $K=k_{0} s_{0}^{2 \kappa} M_{\mathrm{p}}^{\psi \psi \kappa}$ \\
\hline $\begin{array}{l}\text { Equilibrium abundance of consumer in two-trophic-level } \\
\text { food chain }\end{array}$ & $\hat{C}_{2}=\frac{r}{a_{\mathrm{c}}}\left(1-\frac{\hat{R}_{2}}{K}\right) \approx \frac{r}{a_{\mathrm{c}}}$ & $\hat{C}_{2}=\frac{m_{0} s_{0}^{\psi}}{e_{0} a_{0}} M_{\mathrm{p}}^{\psi(\mu-\varepsilon-\alpha)}$ \\
\hline $\begin{array}{l}\text { Equilibrium abundance of consumer in three-trophic-level } \\
\text { food chain }\end{array}$ & $\hat{C}_{3}=\frac{m_{\mathrm{p}}}{e_{\mathrm{p}} a_{\mathrm{p}}}$ & $\hat{C}_{3}=\frac{m_{0}}{e_{0} a_{0}} M_{\mathrm{p}}^{\mu-\varepsilon-\alpha}$ \\
\hline Interaction strength between trophic levels 1 and 2 & $\mathrm{IS}_{12}=\frac{K}{\hat{R}_{2}}=\frac{e_{\mathrm{c}} a_{\mathrm{c}} K}{m_{\mathrm{c}}}$ & $\mathrm{IS}_{12}=\frac{e_{0} a_{0} k_{0} s_{0}^{\kappa+\psi(\varepsilon+\alpha-\mu+\kappa \psi)}}{m_{0}} M_{\mathrm{p}}^{\psi(\varepsilon+\alpha-\mu+\kappa \psi)}$ \\
\hline Interaction strength between trophic levels 2 and 3 & $\mathrm{IS}_{23}=\frac{\hat{C}_{2}}{\hat{C}_{3}}$ & $\mathrm{IS}_{23}=s_{0}^{\psi} M_{\mathrm{p}}^{(\psi-1)(\mu-\varepsilon-\alpha)}$ \\
\hline
\end{tabular}

Note: Parameter scaling functions are given in table 1.

$\mathrm{TC}=\hat{R}_{4} / \hat{R}_{2}$ or $\mathrm{TC}=\hat{R}_{5} / \hat{R}_{2}$ might alter our model predictions. We simplify this extension by assuming that mortality of a consumer due to factors other than predation $\left(m_{\mathrm{c}}\right)$ is much lower than that caused by predation, so that $m_{\mathrm{c}} \approx 0$ for all intermediate trophic levels. With this assumption, in all chains with three or more trophic levels the equilibrium density of the resource is

$$
\hat{R}_{n}=\frac{a_{\mathrm{p}} \hat{P}_{n}}{e_{\mathrm{c}} a_{\mathrm{c}}}
$$

where $n$ is the length of the food chain. Noting that the equilibrium abundance of the predator $\hat{P}_{3}=e_{\mathrm{c}} a_{\mathrm{c}} \hat{R}_{3} / a_{\mathrm{p}}$, we can substitute $a_{\mathrm{p}} / e_{\mathrm{c}} a_{\mathrm{c}}=\hat{R}_{3} / \hat{P}_{3}$ into the expression for $\hat{R}_{n}$, and the trophic cascade expression becomes TC $=$ $\hat{R}_{3} \hat{P}_{n} / \hat{R}_{2} \hat{P}_{3}$, or

$$
\mathrm{TC}_{\mathrm{IS}}=\left(\mathrm{IS}_{12}-\frac{\left(\mathrm{IS}_{12}-1\right)}{\mathrm{IS}_{23}}\right) \frac{\hat{P}_{n}}{\hat{P}_{3}} .
$$

In a three-level food chain, this expression reduces to equation (7) because $\hat{P}_{n}=\hat{P}_{3}$. Thus, in any food chain with more than three levels, the net effect of predators above the predator in trophic level 3 is to modify the strength of the trophic cascade relative to what is set by $\mathrm{IS}_{12}$ and $\mathrm{IS}_{23}$, allowing for the different effect of odd- and evenlength food chains on the trophic cascade. This is also why there is no effect of predator abundance in equation (7), as it matters only when it is modified by trophic interactions at higher levels.

\section{Modeling the Body Size Dependence of the Strength of the Trophic Cascade}

We have now deconstructed the strength of a trophic cascade into its component parts and shown how interaction strengths along the food chain influence the strength of the overall trophic cascade. We now address question 2: how does body size influence the strength of trophic cascades? This question is motivated by the empirical work of Borer et al (2005), Jochum et al. (2012), and Simonis (2013). We will focus on cross-species variation in body size, although similar body size-dependent processes may occur for predators that alter foraging behaviors as they grow in size (Simonis 2013).

We model each equilibrium abundance in equation (6) with size-based expressions described in tables 1 and 2, as has been done previously in other allometric consumerresource models (Yodzis and Innes 1992; Weitz and Levin 2006; DeLong and Vasseur 2012a, 2012b; Pawar et al. 2012). In short, each parameter in the food chain model can be written as a power law function of resource, consumer, or predator body size (table 1). The intrinsic rate of growth and carrying capacity are functions of resource body size $\left(M_{\mathrm{r}}\right)$, while the remaining parameters are functions of consumer $\left(M_{\mathrm{c}}\right)$ and predator $\left(M_{\mathrm{p}}\right)$ body size.

An important step is to link the size of the predator with the size of the consumer and the resource via an additional power law function describing prey size selection (table 1). This is accomplished by recognizing that predators consume individuals within a certain size class 
(Brose et al. 2006; Barnes et al. 2010; Riede et al. 2011; DeLong and Vasseur 2012a, 2012b), which means that the body size of the basal resource can be modeled as a function of the consumer's body size. Here, we do this with a power function: $M_{\mathrm{r}}=s_{0} M_{\mathrm{c}}^{\psi}$. Likewise, we model the body size of the consumer as a function of the body size of the top predator, $M_{\mathrm{c}}=s_{0} M_{\mathrm{p}}^{\psi}$. The functions that relate body size at one trophic level to body size at another are powerful in that they allow all of the parameters of one trophic level to be rescaled relative to a change in body size in the other trophic level. For example, resource-carrying capacity $(K)$ is related to the body size of the basal resource but can be reexpressed in terms of the predator's body size (DeLong and Vasseur 2012a, 2012b). We use this relationship to show how trophic cascades, which are dependent on interactions between all consumer-prey pairs (eq. [7]), can be expressed in terms of predator body size. Predator body size is thus a proxy for the body size of the entire trophic chain, allowing us to compare trophic cascades in chains composed of large organisms to those in chains composed of small organisms. For simplicity, we assume that the allometric functions for area of capture, conversion efficiency, mortality rate, and prey size selection have the same allometric slope and intercept for the consumer and the predator. This assumption is plausible in many situations, such as when food chains consist of taxa that are related (we demonstrate that this is true for protists below), but other cases may produce different outcomes (see "Discussion").

Rather than writing out the full TC as a function of body size, we calculate the body size dependencies of the equilibrium abundances and the two interaction strengths from which the trophic cascade can be calculated (table 2). We show that $\mathrm{IS}_{12}$ is expected to change with predator body size whenever $\varepsilon+\alpha+\kappa \Psi \neq \mu$, whereas IS $_{23}$ is expected to change only if $\Psi \neq 1$ and $\varepsilon+\alpha \neq \mu$. This last inequality also implies that unless carrying capacity is independent of body size (i.e., $\kappa=0$ ), TC must change as a function of body size. It should be noted that although the equilibrium abundances can be written as power law functions of the size of the predator, equation (7) shows that the overall trophic cascade only approaches a power law function of the predator's body size as the strength of $\mathrm{IS}_{23}$ increases.

\section{Two Empirical Case Studies in Size- Structured Trophic Systems}

To identify which trophic cascade component contributes to the overall body size dependence, we parameterized our model using two independent families of scaling parameters. The first is a set of "canonical" parameters, which are thought to hold generally for many groups of organ- isms. Although the canonical scalings may be broadly applicable, it is unknown whether any specific set of food chains actually displays these exact parameter scalings and whether the net effect of body size on the trophic cascade might differ from these canonical expectations. We therefore assembled a second set of parameters from empirical data for protist-protist-algae food chains. For both case studies, we calculate the strength of a trophic cascade across a range of body sizes given the body size dependence of all parameters. We emphasize that we are not testing our model with these scalings but rather are using them as inputs to our model to explore how the body size dependence of trophic interactions can influence the strength of trophic cascades.

\section{Case Study 1: Canonical Scaling Parameters}

Because values of the allometric scaling exponents may vary widely (table 1), we used a set of standard expectations that we refer to as canonical scaling parameters. Across many species, the scaling exponent for prey size with predator size $(\psi)$ is $\approx 1$ (Brose et al. 2006), meaning that the ratio of predator to prey body size remains constant even as body sizes increase. The scaling of area of capture $(\alpha)$ has been thought to parallel that of metabolic rate so that intake rates keep pace with metabolic requirements, suggesting $\alpha \approx 0.75$ (Yodzis and Innes 1992). Mortality rates generally scale as $\mu \approx-0.25$ (McCoy and Gillooly 2008 ), and the scaling of population abundance is often $\kappa \approx-0.75$ (Damuth 1981). Finally, the scaling of efficiency $(\varepsilon)$ has not been empirically determined across a wide range of sizes, but because it depends on the ratio of resource size to consumer size it will be 0 when the scaling exponent for prey size with predator size is 1 (Weitz and Levin 2006; DeLong 2011; Pawar et al. 2012). We do not assess the effect of potential error in these scaling parameters because the parameters do not apply to any specific group of organisms and because error distributions for the canonical expectations are unknown.

\section{Case Study 2: Algae, Grazing Protists, and Carnivorous Protists}

We collated observations on all the parameters composing the food chain model for carnivorous protists consuming protists that graze algae (data are available from the Dryad Digital Repository: http://dx.doi.org/10.5061 /dryad.74854; DeLong et al. 2014a). For trophic levels 1 and 2, we used existing data on the body size dependence of protists grazing on algae that included observations on 44 pairwise interactions (DeLong and Vasseur 2012b). We then assembled a new data set for trophic levels 2 and 3 by including carnivorous protists feeding on other 

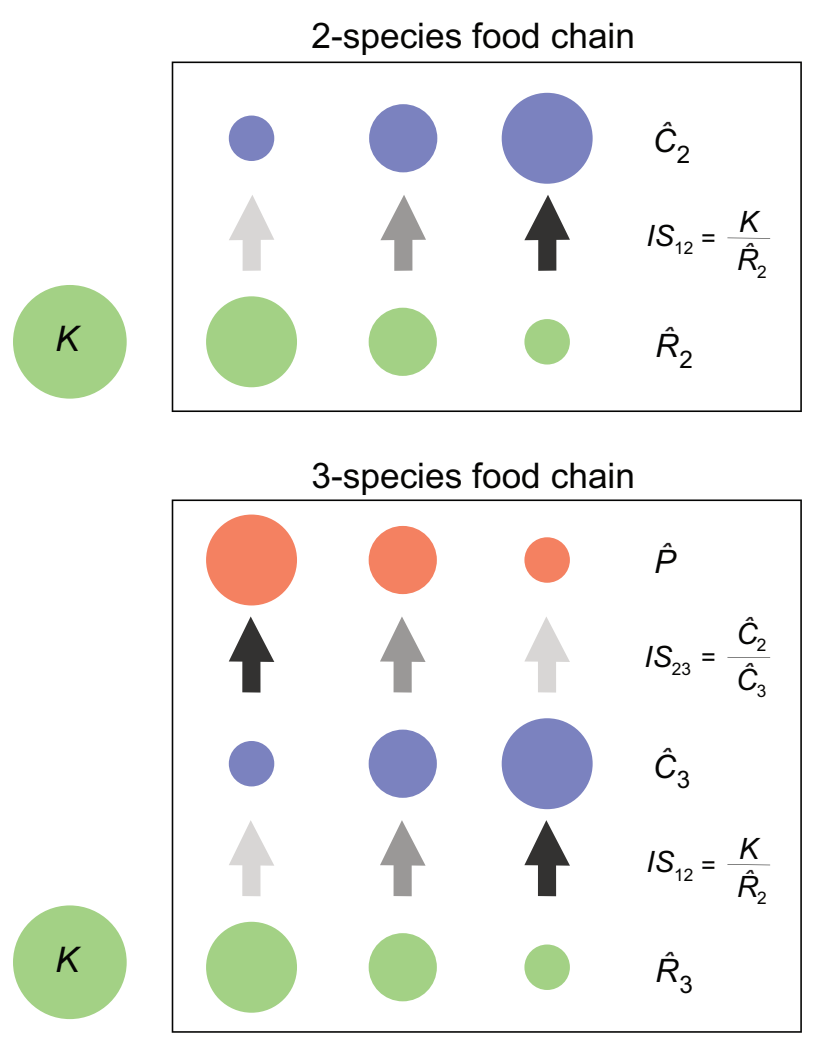

Figure 1: Mechanistic depiction of a trophic cascade as formalized in our model. Circle size denotes relative equilibrium population abundances of the resource (green), consumer (blue), and predator (red), and darkness of arrows denotes trophic interaction strength (IS; darker arrows represent stronger IS). In a two-species food chain (top), the interaction strength between the resource and the consumer (IS $\left.)_{12}\right)$ is the ratio $K / \hat{R}_{2}$. As the equilibrium abundance of the consumer $\left(\hat{C}_{2}\right)$ declines, the equilibrium abundance of the resource $\left(\hat{R}_{2}\right)$ moves back toward carrying capacity $(K)$. In a three-species food chain (bottom), the interaction strength between the predator and the consumer $\left(\mathrm{IS}_{23}\right)$ is the ratio $\hat{C}_{2} / \hat{C}_{3}$. As this ratio gets larger, $\hat{R}_{3}$ moves closer and closer to $K$. Thus, the potential strength of the trophic cascade is set by $\mathrm{IS}_{12}$, and the degree to which that potential is reached is set by $\mathrm{IS}_{23}$.

protists. We identified studies for the carnivorous protist data set by searching Google Scholar for various combinations of "protist," "functional response," and specific genera of carnivorous protists. We also searched the references of other studies of functional responses of protists and the websites of researchers specialized in protist foraging ecology. Last, we perused papers on the foraging ecology of protists where additional such data might exist but not be locatable with a keyword search. This search produced data on 18 pairwise interactions for carnivorous protists (Laybourn 1976; Hewett 1980; Rogerson 1981; Verity 1991; Jeong et al. 2004, 2007a, 2007b; DeLong et al. 2014b). Data on functional response pa- rameters were used as reported if a functional and/or numerical response was fit to foraging data in units of individuals (i.e., as opposed to amounts of carbon). Otherwise, data were digitized, converted to the appropriate units, and refit using ordinary least squares (OLS) regression in Matlab (ver. 2012b). We assumed that the area of capture parameter (a) from a type 2 functional response is a reasonable approximation to that for a type 1 functional response because it reflects the rising, linear part of a functional response. In combination, these scaling data allow us to assess the body size dependence of $\mathrm{IS}_{12}$ (protists grazing algae) and $\mathrm{IS}_{23}$ (carnivorous protists consuming grazing protists). With these new estimates, we can predict the strength of trophic cascades for protists as a case study, although empirical observations to test these predictions are not available. Data on algae growth rates are from Tang (1995), and those on algaecarrying capacity are from DeLong and Vasseur (2012b).

Following DeLong and Vasseur $(2012 a, 2012 b)$, we used both OLS and reduced major axis regression (RMA) to determine the body size dependence of all underlying model parameters. We averaged the parameter estimates from both approaches because OLS regression assumes that body size (the independent variable) is both controlled and known, whereas RMA assumes that the error in the dependent and independent variables are the same by minimizing perpendicular deviations of data from the best-fit line. The real scaling parameters lie somewhere between the parameters produced by OLS and RMA fitting approaches because rate measurements likely have more error than size measurements; therefore, we take the average of the two fitting procedures. We used a bootstrapping procedure (DeLong and Vasseur 2012a) to assess the scaling of each parameter with 10,000 samples with replacement to get the median scaling estimate (50\% quantile) and the $95 \%$ confidence intervals $(2.5 \%$ and $97.5 \%$ quantiles). We first assessed the scaling parameters for grazing and carnivorous protists separately; as they were not statistically different (95\% confidence intervals overlapped in all cases), we fit the data for all protists together (table 1).

We used a full uncertainty analysis with the bootstrapped distributions for each parameter to assess the body size dependence of the equilibrium abundances of the consumer and the resource in both the two-species and the three-species model. That is, we randomly drew parameters from each parameter's bootstrapped distribution 1,000 times for each of a range of body sizes and then used the median along with the $2.5 \%$ and $97.5 \%$ quantiles of the resulting distributions to determine the confidence intervals. 

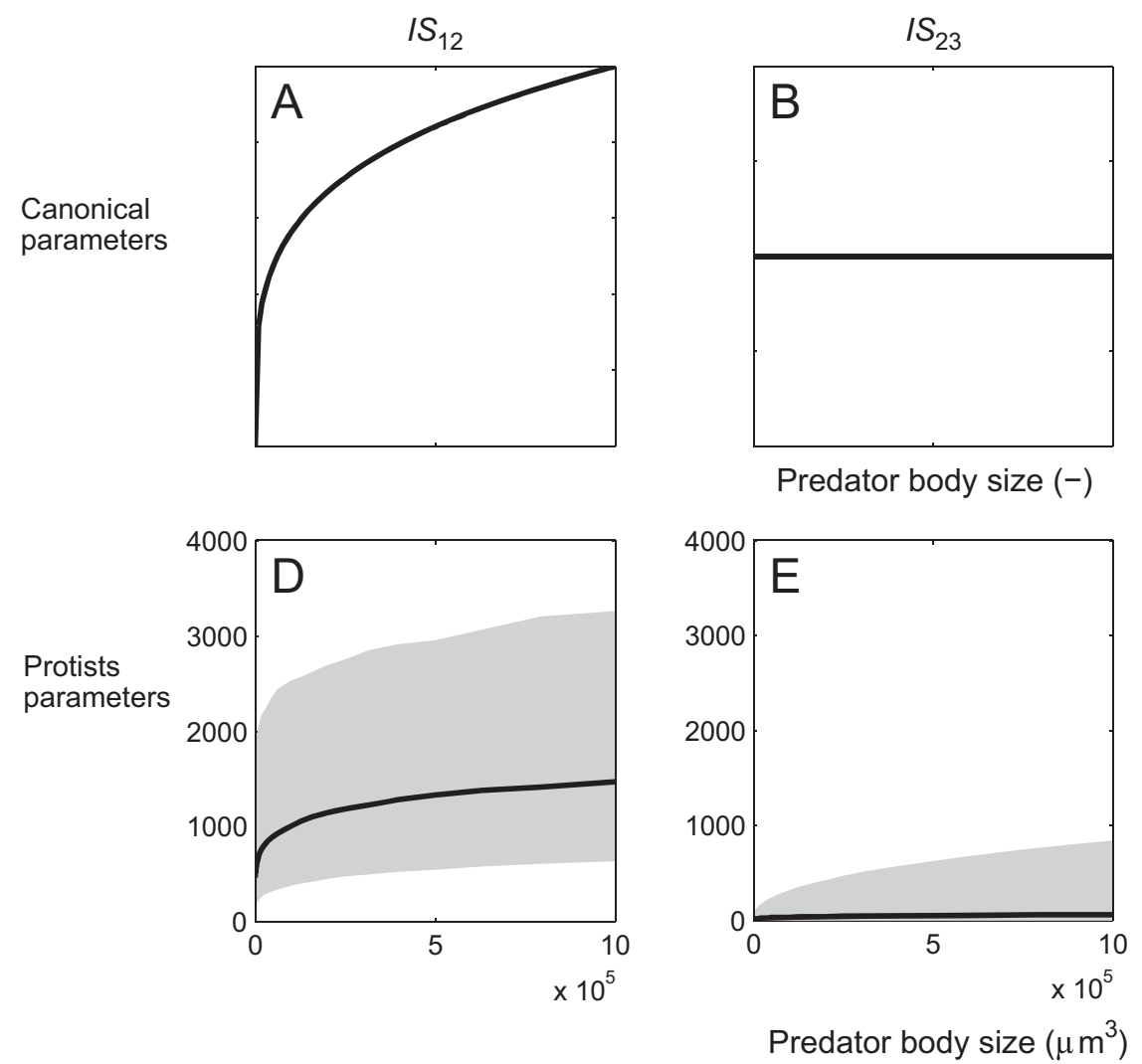

Trophic cascade
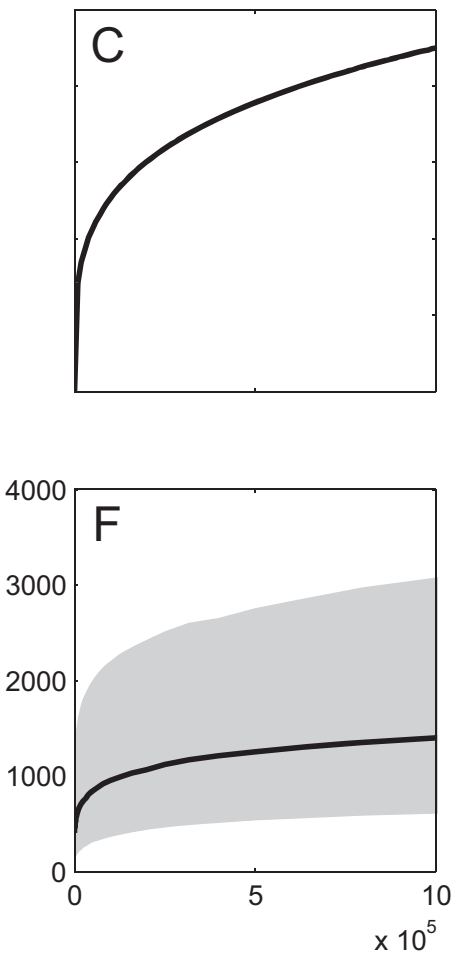

Figure 2: Body size dependence of interaction strengths and trophic cascades. The top row shows predator body size dependence generated with canonical parameters (written without scales because the scaling intercepts are arbitrary), and the bottom row shows empirical body size dependence (with 95\% confidence intervals [gray areas]) for protist food chains. The interaction strength between trophic levels 1 and $2\left(\mathrm{IS}_{12}\right)$ is shown in $A$ and $D$, the interaction strength between trophic levels 2 and $3\left(\mathrm{IS}_{23}\right)$ is shown in $B$ and $E$, and the trophic cascade strength, which is a function of $\mathrm{IS}_{12}$ and $\mathrm{IS}_{23}$, is shown in $C$ and $F$. Both sets of parameters show a clear size dependence of trophic cascades that is generated mostly in $\mathrm{IS}_{12}$.

\section{Results}

\section{Theoretical Consequences of Modeling Trophic Cascades in Terms of Interaction Strengths}

Equation (7) shows that the strength of a trophic cascade depends on two aspects of the three-species interaction (fig. 1). First, an upper limit to the strength of the trophic cascade is set by the interaction between the resource and the consumer $\left(\mathrm{IS}_{12}\right)$. This makes intuitive sense because the resource in the presence of a predator cannot rebound beyond its original carrying capacity: $\mathrm{TC}=\hat{R}_{3} / \hat{R}_{2} \leq$ $K / \hat{R}_{2}$. The trophic cascade approaches the upper limit as the interaction strength between the consumer and predator $\left(\mathrm{IS}_{23}\right)$ gets large, reducing the size of the second term in equation (7). This means that the more the predator reduces the consumer's abundance, the more the resource can increase toward its carrying capacity (fig. 1). The lower limit to the trophic cascade is 1 , and this occurs when either of the interaction strengths reduces to 1 (i.e., when a predator does not affect the resource). Thus, the strength of a trophic cascade occurs in the interval $\left[1, K / \hat{R}_{2}\right]$.

\section{Sensitivity of the Trophic Cascade Strength to Size for Systems with Canonical Scaling Parameters}

Given canonical scaling parameters (table 1), we expect that $\mathrm{IS}_{12}$ in a three-trophic-level system scales with predator body size with a one-fourth power: $\mathrm{IS}_{12} \propto M_{\mathrm{p}}^{\psi(\varepsilon+\alpha-\mu+\kappa \psi)} \propto$ $M_{\mathrm{p}}^{1(0+0.75+0.25-0.75 \cdot 1)} \propto M_{\mathrm{p}}^{0.25}$ (fig. $2 A$ ). In contrast, IS $_{23}$ will be independent of predator body size, given canonical scaling exponents: $\mathrm{IS}_{23} \propto M_{\mathrm{p}}^{(\psi-1)(\mu-\varepsilon-\alpha)} \propto M_{\mathrm{p}}^{(1-1)(-0.25-0-0.75)} \propto M_{\mathrm{p}}^{0}$ (fig. 2B). Thus, as a constant in the scenario where canonical values apply, $\mathrm{IS}_{23}$ can modify the body size dependence set by $\mathrm{IS}_{12}$ but is itself not affected by the size of the predator (fig. $2 C$ ). This suggests that the body size dependence of trophic cascades arises primarily in the interaction between resource and consumer. 

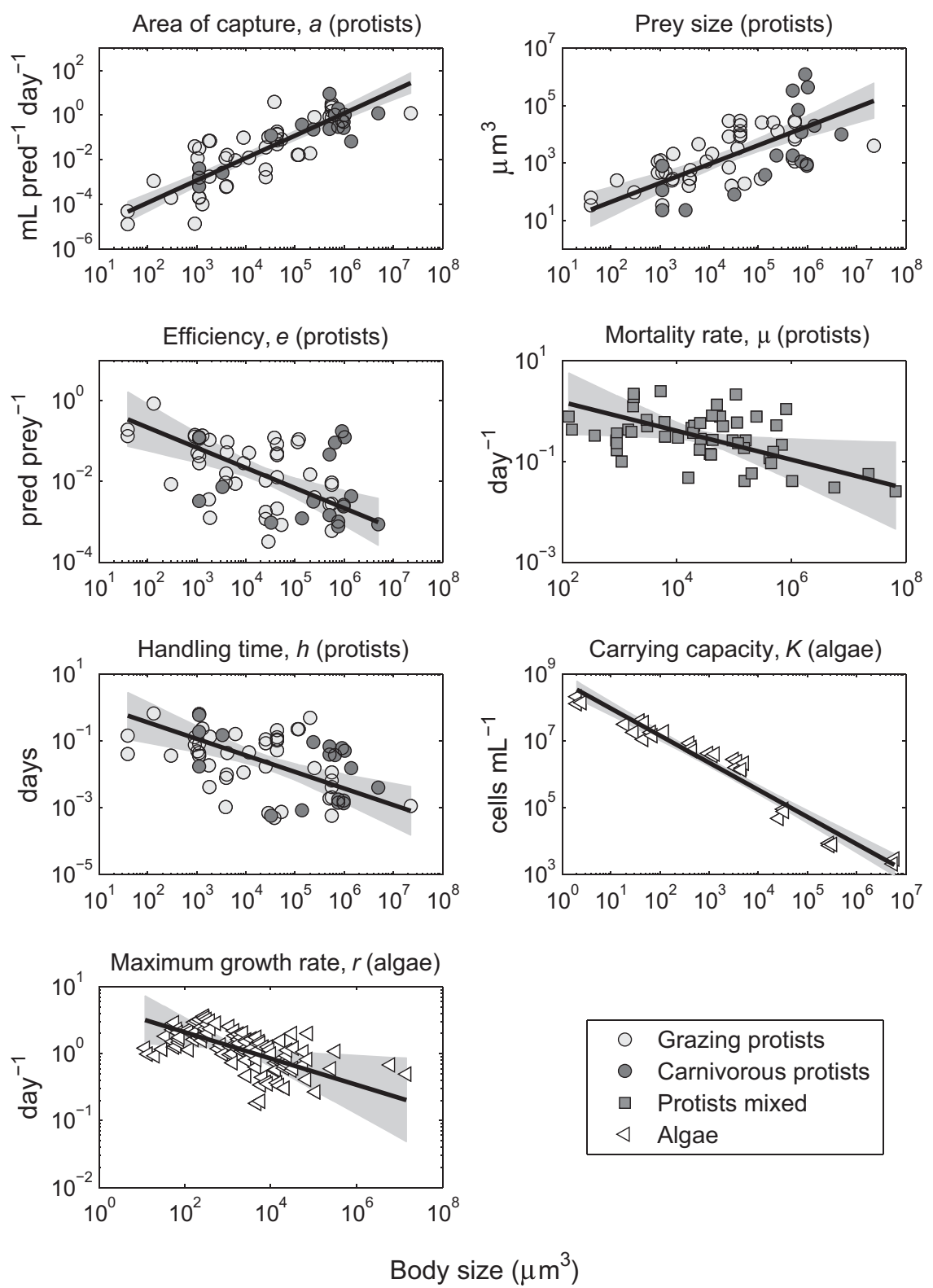

Figure 3: Scaling relationships between model parameters and cell volume for grazing and carnivorous protists and algae. Power law fits are not statistically distinguishable between grazers and carnivores and so are fit together. See table 1 for parameter values. Gray areas indicate $95 \%$ confidence intervals.

\section{Sensitivity of the Trophic Cascade Strength for Protists and Algae}

Body size was a good predictor of protist and algae parameters (fig. 3; table 1). All consumer and resource equilibrium abundances declined with body size of the predator (fig. 4). It is the difference in the slopes of these abundance relationships that drive the body size dependence of interaction strengths, since they are defined as the ratio of abundances $\left(K / \hat{R}_{2}\right.$ and $\left.\hat{C}_{2} / \hat{C}_{3}\right)$. For protists, we predict the interaction strength between the consumer and resource $\left(\mathrm{IS}_{12}\right)$ will increase with predator body size. The slope of the body size dependence is somewhat shallower than the canonical prediction given above (fig. $2 D$ ). In contrast to the case with canonical parameters, there is a very slight dependence of the $\mathrm{IS}_{23}$ on predator body size in protists (fig. $2 E$ ). Because IS $\mathrm{I}_{12}$ and IS $\mathrm{IS}_{23}$ are both dependent on predator body size in protists, there is also a significant positive relationship between predator body size 

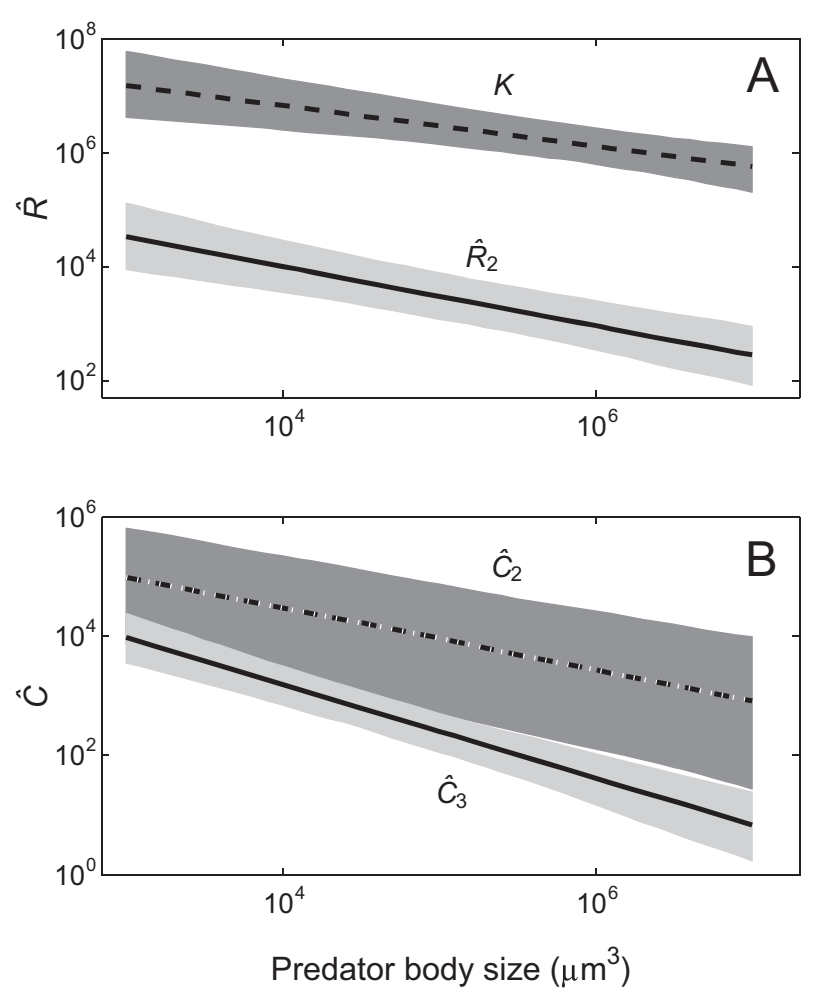

Figure 4: Scaling of equilibrium abundance with predator cell volume for resource populations with $\left(\hat{R}_{2}\right)$ and without $(K)$ a consumer (one- and two-trophic-level food chains; $A$ ) and consumer populations with $\left(\hat{C}_{3}\right)$ and without $\left(\hat{C}_{2}\right)$ a predator $(B)$. Recall that species are linked through prey size selection functions (table 1). Gray areas indicate $95 \%$ confidence intervals.

and the strength of a trophic cascade in this group (fig. $2 F$ ). Thus, for both the canonical scalings and those determined for a large data set derived from real organisms, the strength of a trophic cascade is predicted to have a significant positive dependence on the size of the top predator, an effect that is generated primarily by the body size dependence of $\mathrm{IS}_{12}$.

\section{Discussion}

Trophic cascades play an important role in transmitting the consequences of human-induced loss of top predators on ecosystems (Terborgh et al. 2001; Estes et al. 2011; Wilmers et al. 2012). The strength of the trophic cascade determines the amount of basal resource reduction by consumers when top predators are removed. Our results are consistent with previous empirical work and suggest that food chains with larger predators (and thus larger consumers and primary producers; Brose et al. 2006) may experience stronger trophic cascades than food chains with smaller predators. This suggests that the loss of large pred- ators should have greater consequences for ecosystems than the loss of smaller predators in otherwise similar systems. Borer et al. (2005) found a body size effect on trophic cascades across marine and terrestrial systems as well as vertebrate and invertebrate taxa, suggesting that body size is an important factor despite the high variation caused by these additional factors. The types of changes that occur following the removal of large predators, such as wolves (Ripple and Beschta 2012), cod (Frank et al. 2005), orcas (Estes et al. 1998), and sharks (Myers et al. 2007), suggest that there are substantial consequences of their removal. In contrast, empirical studies as well as our theoretical work suggest that smaller predators generate weak or no trophic cascades (Elser et al. 1998; Meserve et al. 2003; Maron and Pearson 2011; Laws and Joern 2013). Given the generally higher risk of extirpation for largebodied organisms (Davidson et al. 2009; Ripple et al. 2014) and the increased sensitivity of large-bodied species to functional extinction (Säterberg et al. 2013), this effect may be important in driving ecological responses to anthropogenic disturbances.

Our findings provide an important mechanistic explanation of size-structured trophic cascades: the potential for top-down control is set by the interaction strength between the first and second trophic levels (van Veen and Sanders 2013), and the magnitude of this effect depends on the interaction strength between the second and third trophic levels (fig. 1). On the surface, this result appears counterintuitive-how would a larger predator increase a trophic cascade through its effect on consumer-resource interactions that occur when the predator is absent? Our results show that this occurs because larger predators eat larger consumers, which in turn eat larger resources. This matching of body sizes across trophic levels was evident in our data (fig. 3) and has been shown to hold generally across species and ecosystems (e.g., Brose et al. 2006; Barnes et al. 2010). Importantly, the scaling of consumer to resource body sizes generates greater interaction strengths at larger consumer and resource sizes, causing trophic systems with larger body sizes overall to experience larger trophic cascades. These findings depend on the body size dependence of the underlying parameters, which could take on different slopes in different systems. The similarity of the outcomes for a fully parameterized taxonomic system (protist and algae parameters) and the broad scalings expected for many taxa (canonical parameters) suggest that these patterns may hold up. Nonetheless, in systems without strong size-structured foraging relationships (such as mammals grazing in grasslands), body size may influence the strength of trophic cascades differently or not at all. Such cases remain to be found.

The predictions we make with our two sets of empirical parameters cannot yet be tested directly because there are 
no empirical data available to evaluate the body size dependence of trophic cascades in protists or any other specific group. For this, data on the abundance and body size of all species in a trophic cascade would be required, with explicit information on variation in body size across a gradient of predator body size. Nonetheless, our results indicate that the body size dependence of trophic cascades found in the meta-analysis of Borer et al. (2005) primarily arose through the body size dependence of the impacts of consumers on their resources $\left(\mathrm{IS}_{12}\right)$, with a smaller contribution from the size dependence of predator suppression of consumers $\left(\mathrm{IS}_{23}\right)$.

Differences between the strength of marine and terrestrial trophic cascades (Shurin et al. 2006) could be related to body size in multiple ways. First, differences could arise if systematic differences exist between these two habitats with respect to body sizes or the relationship between predator body size and the size of prey that can be consumed. However, differences in body size might be important only if all the underlying allometric scaling parameters are equivalent across habitats. Second, systemwide differences in interaction strengths, in particular $\mathrm{IS}_{12}$, could alter the strength of the trophic cascade via several underlying parameters, including carrying capacity, predator mortality rate, area of capture, and conversion efficiency (see tables $1,2)$. Clearly, systematic aggregate differences in the predator-prey interactions among ecosystems need to be assessed (Gilbert et al. 2014) and are likely to arise due to taxonomic and metabolic differences between habitats (Shurin and Seabloom 2005), stoichiometric nutritional quality (Elser et al. 2000; Hall et al. 2007), and predatorprey size ratios (Shurin and Seabloom 2005; Brose et al. 2006). Finally, differences in food chain length (Hairston and Hairston 1993), especially when related to the size of the top predator, could alter the strength of trophic cascades across habitat types.

Recently, Pawar et al. (2012) showed how the dimensionality of the searching environment could alter the area of capture scaling as the units of this parameter change from area per predator per time to volume per predator per time. Thus, we might expect the canonical scalings to vary with searching dimensions. In 2-D environments the body size scaling for area of capture is estimated to be 0.63 , and in 3-D environments it is estimated to be 1.03 (Pawar et al. 2012), suggesting that the body size dependence of a trophic cascade should be reduced in 2-D relative to 3-D environments. The body size dependence for the interaction strength between the first and second trophic levels ( $\mathrm{IS}_{12}$, where the body size dependence of the trophic cascade lies in the canonical scenario) is thus predicted to be 0.13 in 2-D environments versus 0.53 in 3$\mathrm{D}$ environments. However, the scaling of resource abundance, a proxy for carrying capacity, also may change with dimensionality, shifting from -0.76 in $2-\mathrm{D}$ to -1.12 in 3-D (Pawar et al. 2012). For example, phytoplankton abundance tends to scale close to linearly with their cell size in both experimental and field settings (DeLong and Vasseur 2012b; Huete-Ortega et al. 2012). These shifts change the scaling of $\mathrm{IS}_{12}$ to 0.13 in $2-\mathrm{D}$ and 0.16 in 3-D. Therefore, a simultaneous change in area of capture and carrying capacity may negate the overall effect of dimensionality on the trophic cascade, although more work is needed to clarify these effects. In any case, stronger cascades in benthic freshwater and marine habitats $(2-D)$ than in their pelagic counterparts (3-D; Micheli 1999; Shurin et al. 2002) suggest that other environmental factors may override the dimensionality effects.

This also brings up the interesting possibility that intercepts and slopes of the underlying parameter scalings may vary across trophic levels. Shifts in the scaling exponents or intercepts across trophic levels may occur when, for example, endotherms are foraging on ectotherms, such as birds eating insects (Dell et al. 2014). Greater similarities may exist in food chains that include similar taxa across levels, as we showed with protists, but also perhaps in planktivorous and carnivorous fish or invertebrate food chains. Fully parameterizing our model for such scenarios, as we have done with protists, requires more data than are usually available for diverse taxa, although it seems likely that additional data sets could be generated for carnivorous fish, mammals, or insects. In addition, there may be challenges in parameterizing our model for trophic interactions where organisms are not consumed whole and so the link between consumer size and resource size is less clear (e.g., herbivorous mammals). Nonetheless, we can illustrate how such changes could arise. For example, different predator taxa of the same size may consume, on average, larger or smaller prey, shifting the prey size selection functions and altering the strength of the trophic cascade. In this scenario, raising or lowering the parameter $s_{0}$ relative to the expected value will alter both $\mathrm{IS}_{12}$ and $\mathrm{IS}_{23}$ and change the strength of the trophic cascade (see table 1).

Our analysis uses classical predator-prey models that do not consider the potential behavioral or physiological changes that are induced in prey by the presence of predators (e.g., Creel et al. 2007). Further changes due to body size could arise if there are systematic changes in the degree to which fear induces demographic responses in prey. For example, if predation risk induces prey defenses, then the area of capture parameter may be depressed relative to the size-based expectation ( $a_{0}$ declines), lowering interaction strengths and the strength of either the potential for a trophic cascade (if affecting IS $_{12}$ ) or its realization (if affecting $\mathrm{IS}_{23}$ ). Because predation risk tends to push demographic rates in the same direction as actual predation 
(Zanette et al. 2011), this effect could be strong. It may be particularly important for the reintroduction of predators if prey have developed altered life histories in the predator's absence. Similarly, the degree to which other indirect effects have a body size component, such as nutrient subsidies (Croll et al. 2005), could further alter our expectations of how body size influences trophic cascades.

Our framework can also be used to assess the temperature dependence of trophic cascades. Although trophic cascades may strengthen with temperature (Barton et al. 2009; Hoekman 2010; Harley 2011; Kratina et al. 2012), given the effects that temperature may have on the underlying parameters (Dell et al. 2011, 2014) and interaction strengths (Gilbert et al. 2014), the temperature dependence of a trophic cascade is likely to be system specific and context dependent (Laws and Joern 2013). Increases in the underlying parameters for ectotherms could raise the intercepts of the parameter scalings, resulting in a stronger trophic cascade. Furthermore, shifts in the scaling relationship between predator and prey body size with temperature may alter the size dependence of the trophic cascade (Gibert and DeLong 2014). In addition, because model parameters depend on both temperature and body size and because body size responds to both temperature and resources (Kimmance et al. 2006), one might expect a "parameter cascade" due to long-term changes in temperature.

In conclusion, we have shown that allometric predatorprey models can be used to predict the body size dependence of trophic cascades, although explicit testing of model predictions is still required. Our results suggest that generally-and specifically for food chains of protiststhe strength of a trophic cascade should increase with the size of the top predator. This effect is generated mostly by the body size dependence of the interaction strength between the first and second trophic positions $\left(\mathrm{IS}_{12}\right)$. Trophic cascades are widespread but highly variable in their strength (Pace et al. 1999; Schmitz et al. 2000; Shurin et al. 2002). With an understanding of how other processes (e.g., temperature) influence the underlying parameters, our approach could be extended to gain further insights into variation in trophic cascades across ecosystems, with key environmental gradients or under a variety of evolutionary scenarios involved in predator harvest, body size evolution, and productivity changes. These developments represent a key advance in our ability to understand and anticipate the ecosystem consequences of variation in predator size.

\section{Acknowledgments}

This article was generated as part of the Canadian Institute of Ecology and Evolution (CIEE) working group "Thermal
Scaling and Body Size: The Next Frontier in Climate Change" and as part of the working group "Synthesizing Theory and Databases to Advance a General Framework for How Warming Affects Trophic Interactions," organized by M.I.O. and H.S.G. and held at the National Center for Ecological Analysis and Synthesis (NCEAS), which is supported by the National Science Foundation (grant EF0553768); the University of California, Santa Barbara; and the state of California. We appreciate the helpful comments of three anonymous reviewers.

\section{Literature Cited}

Atwood, T. B., E. Hammill, H. S. Greig, P. Kratina, J. B. Shurin, D. S. Srivastava, and J. S. Richardson. 2013. Predator-induced reduction of freshwater carbon dioxide emissions. Nature Geoscience 6:191-194.

Barnes, C., D. Maxwell, D. C. Reuman, and S. Jennings. 2010. Global patterns in predator-prey size relationships reveal size dependency of trophic transfer efficiency. Ecology 91:222-232.

Barton, B. T., A. P. Beckerman, and O. J. Schmitz. 2009. Climate warming strengthens indirect interactions in an old-field food web. Ecology 90:2346-2351.

Beddington, J. R. 1975. Mutual interference between parasites or predators and its effect on searching efficiency. Lournal of Animal Ecology 44:331-340.

Berlow, E. L., A.-M. Neutel, J. E. Cohen, P. C. De Ruiter, B. Ebenman, M. Emmerson, J. W. Fox, et al. 2004. Interaction strengths in food webs: issues and opportunities. Journal of Animal Ecology 73:585598.

Borer, E. T., E. W. Seabloom, J. B. Shurin, K. E. Anderson, C. A. Blanchette, B. Broitman, S. D. Cooper, et al. 2005. What determines the strength of a trophic cascade? Ecology 86:528-537.

Brose, U., T. Jonsson, E. L. Berlow, P. Warren, C. Banasek-Richter, L.-F. Bersier, J. L. Blanchard, et al. 2006. Consumer-resource bodysize relationships in natural food webs. Ecology 87:2411-2417.

Burkepile, D. E. 2013. Comparing aquatic and terrestrial grazing ecosystems: is the grass really greener? Oikos 122:306-312.

Carpenter, S., J. Kitchell, and J. Hodgson. 1985. Cascading trophic interactions and lake productivity. BioScience 35:634-639.

Chase, J. M. 2000. Are there real differences among aquatic and terrestrial food webs? Trends in Ecology and Evolution 15:408412.

Costamagna, A. C., D. A. Landis, and C. D. Difonzo. 2007. Suppression of soybean aphid by generalist predators results in a trophic cascade in soybeans. Ecological Applications 17:441-451.

Creel, S., D. Christianson, S. Liley, and J. A. Winnie. 2007. Predation risk affects reproductive physiology and demography of elk. $\underline{\text { Sci- }}$ ence 315:960.

Croll, D. A., J. L. Maron, J. A. Estes, E. M. Danner, and G. V. Byrd. 2005. Introduced predators transform subarctic islands from grassland to tundra. Science 307:1959-1961.

Damuth, J. 1981. Population density and body size in mammals. Nature 290:699-700.

Davidson, A. D., M. J. Hamilton, A. G. Boyer, J. H. Brown, and G. Ceballos. 2009. Multiple ecological pathways to extinction in mammals. Proceedings of the National Academy of Sciences of the USA 106:10702-10705. 
DeAngelis, D. L., R. A. Goldstein, and R. V. O’Neill. 1975. A model for trophic interaction. Ecology 56:881-892.

Dell, A. I., S. Pawar, and V. M. Savage. 2011. Systematic variation in the temperature dependence of physiological and ecological traits. Proceedings of the National Academy of Sciences of the USA 108:10591-10596.

- 2014. Temperature dependence of trophic interactions are driven by asymmetry of species responses and foraging strategy. Journal of Animal Ecology 83:70-84.

DeLong, J. P. 2011. Energetic inequivalence in eusocial insect colonies. Biology Letters 7:611-614.

DeLong, J. P., B. Gilbert, J. B. Shurin, V. M. Savage, B. T. Barton, C. F. Clements, A. I. Dell, et al. 2014a. Data from: The body size dependence of trophic cascades. American Naturalist, Dryad Digital Repository, http://dx.doi.org/10.5061/dryad.74854.

DeLong, J. P., T. C. Hanley, and D. A. Vasseur. 2014b. Predator-prey dynamics and the plasticity of predator body size. Functional Ecology 28:487-493.

DeLong, J. P., and D. A. Vasseur. 2012a. A dynamic explanation of size-density scaling in carnivores. Ecology 93:470-476.

- 2012b. Size-density scaling in protists and the links between consumer-resource interaction parameters. Iournal of Animal Ecology 81:1193-1201.

Elser, J. J., T. H. Chrzanowski, R. W. Sterner, and K. H. Mills. 1998. Stoichiometric constraints on food-web dynamics: a whole-lake experiment on the Canadian Shield. Ecosystems 1:120-136.

Elser, J. J., W. F. Fagan, R. F. Denno, D. R. Dobberfuhl, A. Folarin, A. Huberty, S. Interlandi, et al. 2000. Nutritional constraints in terrestrial and freshwater food webs. Nature 408:578-580.

Emmerson, M., J. M. Montoya, and G. Woodward. 2006. Body size, interaction strength, and food web dynamics. Pages 167-178 in P. de Ruiter, V. Wolters, J. Moore, and K. Melville-Smith. Dynamic food webs. Elsevier, Amsterdam.

Emmerson, M. C., and D. Raffaelli. 2004. Predator-prey body size, interaction strength and the stability of a real food web. $\underline{\text { Journal }}$ of Animal Ecology 73:399-409.

Estes, J. A., J. Terborgh, J. S. Brashares, M. E. Power, J. Berger, W. J. Bond, S. R. Carpenter, et al. 2011. Trophic downgrading of planet Earth. Science 333:301-306.

Estes, J. A., M. T. Tinker, T. M. Williams, and D. F. Doak. 1998. Killer whale predation on sea otters linking oceanic and nearshore ecosystems. Science 282:473-476.

Frank, K. T., B. Petrie, J. S. Choi, and W. C. Leggett. 2005. Trophic cascades in a formerly cod-dominated ecosystem. Science 308: 1621-1623.

Frank, K. T., B. Petrie, N. L. Shackell, and J. S. Choi. 2006. Reconciling differences in trophic control in mid-latitude marine ecosystems. Ecology Letters 9:1096-1105.

Gibert, J. P., and J. P. DeLong. 2014. Temperature alters food web body-size structure. Biology Letters 10:20140473.

Gilbert, B., T. D. Tunney, K. S. McCann, J. P. DeLong, D. A. Vasseur, V. Savage, J. B. Shurin, et al. 2014. A bioenergetic framework for the temperature dependence of trophic interactions. Ecology Letters 17:902-914.

Hairston, N. G., F. E. Smith, and L. B. Slobodkin. 1960. Community structure, population control, and competition. American Naturalist 94:421-425.

Hairston, N. G., Jr., and N. G. Hairston Sr. 1993. Cause-effect relationships in energy flow, trophic structure, and interspecific interactions. American Naturalist 142:379-411.
Hall, S. R., J. B. Shurin, S. Diehl, and R. M. Nisbet. 2007. Food quality, nutrient limitation of secondary production, and the strength of trophic cascades. Oikos 116:1128-1143.

Harley, C. D. G. 2011. Climate change, keystone predation, and biodiversity loss. Science 334:1124-1127.

Hewett, S. W. 1980. The effect of prey size on the functional and numerical responses of a protozoan predator to its prey. Ecology 61:1075-1081.

Hoekman, D. 2010. Turning up the heat: temperature influences the relative importance of top-down and bottom-up effects. Ecology 91:2819-2825.

Hrbacek, J., M. Dvorakova, V. Korinek, and L. Prochazkova. 1961. Demonstration of the effect of the fish stock on the species composition of zooplankton and the intensity of metabolism of the whole plankton assemblage. Verhandlungen der Internationalen Vereinigung für Theoretische und Angewandte Limnologie 14: 192-195.

Huete-Ortega, M., P. Cermeño, A. Calvo-Díaz, and E. Marañón. 2012. Isometric size-scaling of metabolic rate and the size abundance distribution of phytoplankton. Proceedings of the Roval Society B: Biological Sciences 279:1815-1823.

Jeong, H. J., J. S. Kim, J. Y. Song, J. H. Kim, T. H. Kim, S. K. Kim, and N. S. Kang. 2007a. Feeding by protists and copepods on the heterotrophic dinoflagellates Pfiesteria piscicida, Stoeckeria algicida, and Luciella masanensis. Marine Ecology Progress Series 349:199211.

Jeong, H. J., J. E. Song, N. S. Kang, S. Kim, Y. D. Yoo, and J. Y. Park. 2007b. Feeding by heterotrophic dinoflagellates on the common marine heterotrophic nanoflagellate Cafeteria sp. Marine Ecology Progress Series 333:151-160.

Jeong, H. J., Y. D. Yoo, J. S. Kim, N. S. Kang, T. H. Kim, and J. H. Kim. 2004. Feeding by the marine planktonic ciliate Strombidinopsis jeokjo on common heterotrophic dinoflagellates. Aquatic Microbial Ecology 36:181-187.

Jochum, M., F. D. Schneider, T. P. Crowe, U. Brose, and E. J. O'Gorman. 2012. Climate-induced changes in bottom-up and topdown processes independently alter a marine ecosystem. Philosophical Transactions of the Roval Society B: Biological Sciences 367:2962-2970.

Kimmance, S. A., D. Atkinson, and D. J. S. Montagnes. 2006. Do temperature-food interactions matter? responses of production and its components in the model heterotrophic flagellate Oxyrrhis marina. Aquatic Microbial Ecology 42:63-73.

Kratina, P., H. S. Greig, P. L. Thompson, T. S. A. Carvalho-Pereira, and J. B. Shurin. 2012. Warming modifies trophic cascades and eutrophication in experimental freshwater communities. Ecology 93:1421-1430.

Laws, A. N., and A. Joern. 2013. Predator-prey interactions in a grassland food chain vary with temperature and food quality. Oikos 122:977-986.

Laybourn, J. 1976. Energy budgets for Stentor coeruleus Ehrenberg (Ciliophora). Oecologia (Berlin) 22:431-437.

Maron, J. L., and D. E. Pearson. 2011. Vertebrate predators have minimal cascading effects on plant production or seed predation in an intact grassland ecosystem. Ecology Letters 14:661-669.

McCoy, M. W., and J. F. Gillooly. 2008. Predicting natural mortality rates of plants and animals. Ecology Letters 11:710-716.

Meserve, P. L., D. A. Kelt, W. B. Milstead, and J. R. Gutiérrez. 2003. Thirteen years of shifting top-down and bottom-up control. BioScience 53:633-646. 
Micheli, F. 1999. Eutrophication, fisheries, and consumer-resource dynamics in marine pelagic ecosystems. Science 285:1396-1398.

Myers, R. A., J. K. Baum, T. D. Shepherd, S. P. Powers, and C. H. Peterson. 2007. Cascading effects of the loss of apex predatory sharks from a coastal ocean. Science 315:1846-1850.

Pace, M. L., J. J. Cole, S. R. Carpenter, and J. F. Kitchell. 1999. Trophic cascades revealed in diverse ecosystems. Trends in Ecology and Evolution 14:483-488.

Paine, R. T. 1980. Food webs: linkage, interaction strength and community infrastructure. Journal of Animal Ecology 49:666-685.

Pawar, S., A. I. Dell, and V. M. Savage. 2012. Dimensionality of consumer search space drives trophic interaction strengths. Nature 486:485-489.

Polis, G. A. 1999. Why are parts of the world green? multiple factors control productivity and the distribution of biomass. Oikos 86:3.

Polis, G. A., and D. R. Strong. 1996. Food web complexity and community dynamics. American Naturalist 147:813-846.

Riede, J. O., U. Brose, B. Ebenman, U. Jacob, R. Thompson, C. R. Townsend, and T. Jonsson. 2011. Stepping in Elton's footprints: a general scaling model for body masses and trophic levels across ecosystems. Ecology Letters 14:169-178.

Ripple, W. J., and R. L. Beschta. 2012. Trophic cascades in Yellowstone: the first 15 years after wolf reintroduction. Biological Conservation 145:205-213.

Ripple, W. J., J. A. Estes, R. L. Beschta, C. C. Wilmers, E. G. Ritchie, M. Hebblewhite, J. Berger, et al. 2014. Status and ecological effects of the world's largest carnivores. Science 343:1241484.

Rogerson, A. 1981. The ecological energetics of Amoeba proteus (Protozoa). Hydrobiologia 85:117-128.

Säterberg, T., S. Sellman, and B. Ebenman. 2013. High frequency of functional extinctions in ecological networks. Nature 499:468-470.

Schmitz, O. J., P. A. Hambäck, and A. P. Beckerman. 2000. Trophic cascades in terrestrial systems: a review of the effects of carnivore removals on plants. American Naturalist 155:141-153.

Shurin, J. B., E. T. Borer, E. W. Seabloom, K. Anderson, C. A. Blanchette, B. Broitman, S. D. Cooper, et al. 2002. A cross-ecosystem comparison of the strength of trophic cascades. Ecology Letters 5: 785-791.

Shurin, J. B., D. S. Gruner, and H. Hillebrand. 2006. All wet or dried up? real differences between aquatic and terrestrial food webs. Proceedings of the Roval Society B: Biological Sciences 273:1-9.

Shurin, J. B., and E. W. Seabloom. 2005. The strength of trophic cascades across ecosystems: predictions from allometry and energetics. Journal of Animal Ecology 74:1029-1038.
Simonis, J. L. 2013. Predator ontogeny determines trophic cascade strength in freshwater rock pools. Ecosphere 4:62.

Strickland, M. S., D. Hawlena, A. Reese, M. A. Bradford, and O. J. Schmitz. 2013. Trophic cascade alters ecosystem carbon exchange. Proceedings of the National Academv of Sciences of the USA 110: 11035-11038.

Tang, E. P. Y. 1995. The allometry of algal growth rates. $\underline{\text { Journal of }}$ Plankton Research 17:1325-1335.

Terborgh, J., and J. A. Estes. 2010. Trophic cascades predators, prey, and the changing dynamics of nature. Island Press, Washington, DC.

Terborgh, J., L. Lopez, P. Nuñez, M. Rao, G. Shahabuddin, G. Orihuela, M. Riveros, et al. 2001. Ecological meltdown in predatorfree forest fragments. Science 294:1923-1926.

Tunney, T. D., K. S. McCann, N. P. Lester, and B. J. Shuter. 2012. Food web expansion and contraction in response to changing environmental conditions. Nature Communications 3:1105.

van Veen, F. J. F., and D. Sanders. 2013. Herbivore identity mediates the strength of trophic cascades on individual plants. Ecosphere 4:64.

Verity, P. G. 1991. Measurement and simulation of prey uptake by marine planktonic ciliates fed plastidic and aplastidic nanoplankton. Limnology and Oceanography 36:729-749.

Vucic-Pestic, O., B. C. Rall, G. Kalinkat, and U. Brose. 2010. Allometric functional response model: body masses constrain interaction strengths. Journal of Animal Ecology 79:249-256.

Weitz, J. S., and S. A. Levin. 2006. Size and scaling of predator-prey dynamics. Ecology Letters 9:548-557.

Wilmers, C. C., J. A. Estes, M. Edwards, K. L. Laidre, and B. Konar. 2012. Do trophic cascades affect the storage and flux of atmospheric carbon? an analysis of sea otters and kelp forests. Frontiers in Ecology and the Environment 10:409-415.

Wootton, J. T., and M. Emmerson. 2005. Measurement of interaction strength in nature. Annual Review of Ecology, Evolution, and Systematics 36:419-444.

Yodzis, P., and S. Innes. 1992. Body size and consumer-resource dynamics. American Naturalist 139:1151-1175.

Zanette, L. Y., A. F. White, M. C. Allen, and M. Clinchy. 2011. Perceived predation risk reduces the number of offspring songbirds produce per year. Science 334:1398-1401.

Associate Editor: Frederick R. Adler Editor: Judith L. Bronstein 


\title{
Appendix from J. P. DeLong et al., "The Body Size Dependence of Trophic Cascades"
}

\author{
(Am. Nat., vol. 185, no. 3, p. 354)
}

\section{Analytical Formulation of the Trophic Cascade Model}

In the main text, we developed an expression for the strength of a trophic cascade. We defined a trophic cascade as $\hat{R}_{3} / \hat{R}_{2}$, which is the ratio of the equilibrium abundance of the basal resource when occurring with its consumer and a predator, $\hat{R}_{3}$, to the equilibrium abundance of a resource when occurring only with its consumer, $\hat{R}_{2}$. Thus, higher values indicate that the resource has rebounded in the presence of a predator to a greater extent. We then substituted into this an expression for $\hat{R}_{3}$ that was obtained by solving a three-trophic-level food chain model. The food chain model used linear type 1 functional responses, which took the form of $f=a_{\mathrm{c}} R$, where $R$ is the resource density and $a_{\mathrm{c}}$ is the area of capture or attack rate. Our resulting model is

$$
\mathrm{TC}=\frac{\hat{R}_{3}}{\hat{R}_{2}}=\frac{K}{\hat{R}_{2}}\left(1-\frac{\hat{C}_{3}\left(1-\left[\hat{R}_{2} / K\right)\right]}{\hat{C}_{2}}\right)
$$

(eq. [6] in the main text). In this expression, the equilibrium abundances of resource $\hat{R}$ and consumer $\hat{C}$ in two- and three-trophic-level models are subscripted 2 and 3, respectively, while $K$ is the resource equilibrium in the resource-only model. Now we show that the use of a saturating type 2 functional response, $f=a_{\mathrm{c}} R /\left(1+a_{\mathrm{c}} h R\right)$, where $h$ is the handling time, gives qualitatively the same answer.

Using a type 1 functional response, we could derive equation (A1) because solving for the equilibrium resource density in the three-trophic-level model was possible:

$$
\hat{R}_{3}=K\left(1-\frac{a_{\mathrm{c}} \hat{C}_{3}}{r}\right) \text {. }
$$

But getting an equivalent expression for $\hat{R}_{3}$ using the models with type 2 functional responses, with $\hat{R}_{3}$ only on the lefthand side, is not possible. Nonetheless, we are able to show that the structure we revealed with equation (A1) holds even when a type 2 functional response is used. An unresolved expression for $\hat{R}_{3}$ using a type 2 functional response is

$$
\hat{R}_{3}=K\left(1-\frac{a_{\mathrm{c}} \hat{C}_{3}}{r\left(1+a_{\mathrm{c}} h_{\mathrm{c}} \hat{R}_{3}\right)}\right),
$$

where $h_{\mathrm{c}}$ is the consumer's handling time (you can see $\hat{R}_{3}$ is still on the right-hand side). Plugging this into equation (A1) gives

$$
\mathrm{TC}=\frac{\hat{R}_{3}}{\hat{R}_{2}}=\frac{K}{\hat{R}_{2}}\left(1-\frac{a_{\mathrm{c}} \hat{C}_{3}}{r\left(1+a_{\mathrm{c}} h_{\mathrm{c}} \hat{R}_{3}\right)}\right) .
$$

Now we solve for $a_{\mathrm{c}} / r$ when the resource equation is set to 0 , using the two-trophic-level model equation for resource dynamics

$$
\frac{\mathrm{d} R}{\mathrm{~d} t}=r R\left(1-\frac{R}{K}\right)-\frac{a_{\mathrm{c}} R C}{1+a_{\mathrm{c}} h_{\mathrm{c}} R}
$$

giving

$$
\frac{a_{\mathrm{c}}}{r}=\frac{\left[1-\left(\hat{R}_{2} / K\right)\right]\left(1+a_{\mathrm{c}} h_{\mathrm{c}} \hat{R}_{2}\right)}{\hat{C}_{2}},
$$

which can be plugged into equation (A2) to get

$$
\mathrm{TC}=\frac{\hat{R}_{3}}{\hat{R}_{2}}=\frac{K}{\hat{R}_{2}}\left(1-\frac{\hat{C}_{3}\left[1-\left(\hat{R}_{2} / K\right)\right]}{\hat{C}_{2}} \frac{\left(1+a_{\mathrm{c}} h_{\mathrm{c}} \hat{R}_{2}\right)}{\left(1+a_{\mathrm{c}} h_{\mathrm{c}} \hat{R}_{3}\right)}\right) .
$$


Equation (A3) shows nearly the same expression for a TC as shown in equation (1), but here the interaction strength between trophic levels 2 and $3\left(\mathrm{IS}_{23}=\hat{C}_{2} / \hat{C}_{3}\right)$ is now modified by the ratio of the denominators of the type 2 functional responses in the two- and three-trophic-level models $\left(\left(1+a_{\mathrm{c}} h_{\mathrm{c}} \hat{R}_{2}\right) /\left(1+a_{\mathrm{c}} h_{\mathrm{c}} \hat{R}_{3}\right)\right)$. If there is no trophic cascade, $\hat{R}_{3}=\hat{R}_{2}$, and the expression collapses to the original (eq. [A1]). If there is a trophic cascade, then $\hat{R}_{3}>\hat{R}_{2}$, and $\mathrm{IS}_{23}$ now has to be smaller than it was without a handling time to keep the expression true for any given TC. What this means is that type 2 functional responses serve to heighten $\mathrm{IS}_{23}$ without altering the fundamental structure we have uncovered in the main text. Furthermore, if we used a type 2 functional response that included other terms in the denominator-such as the Beddington-DeAngelis functional response, which includes a wasted-time term $w$ for interference $\left(f=a_{\mathrm{c}} R /\left(1+w(C-1)+a_{\mathrm{c}} h R\right)\right.$; Beddington 1975; DeAngelis et al. 1975) - the outcome is the same because everything in the denominator gets carried through to the ratio of denominators in equation (A3).

Given that $\mathrm{IS}_{23}$ should get smaller in the presence of a nonzero handling time in order to maintain the same strength of trophic cascade with a nonzero handling time, it is useful to understand if this is indeed the case as our model transitions from a type 1 to type 2 functional response. Although it is not possible to obtain a solution for $\hat{R}_{3}$ in the model with type 2 functional responses, $\hat{C}_{2}$ and $\hat{C}_{3}$ are both analytically tractable, allowing us to write an expression for $\mathrm{IS}_{23}$ :

$$
\mathrm{IS}_{23}=\frac{\hat{C}_{2}}{\hat{C}_{3}}=\frac{\left(r / a_{\mathrm{c}}\right)\left[1-\left(\hat{R}_{2} / K\right)\right]\left(1+a_{\mathrm{c}} h_{\mathrm{c}} \hat{R}_{2}\right)}{m_{\mathrm{p}} /\left[a_{\mathrm{p}}\left(e_{\mathrm{p}}-h_{\mathrm{p}} m_{\mathrm{p}}\right)\right]} .
$$

When $h_{\mathrm{c}}$ and $h_{\mathrm{p}}$ are equal to 0 , this expression reduces to the type 1 model in the main text. Focusing on the condition that defines the transition from type 1 to type 2 models, we take the derivative of this expression with respect to handling time (which is 0 in type 1 and positive in type 2) and solve this expression when $h=0$. The sign of this expression is determined by

$$
1-\frac{e_{\mathrm{c}} m_{\mathrm{p}}}{e_{\mathrm{p}} m_{\mathrm{c}}}-\frac{m_{\mathrm{c}}}{a_{\mathrm{c}} e_{\mathrm{c}}\left(K-\hat{R}_{2}\right)} .
$$

Under the assumption that consumers and predators have similar conversion efficiencies and death rates, the value of the second term is near -1 . For the case of protists in the main text, the smaller consumer will have a higher efficiency than the predator and a higher mortality rate, thus creating two ratios that should multiply out to be close to 1 . Because the third term is always negative, IS $_{23}$ will therefore decline as the functional responses shift from type 1 to type 2 . However, If $e_{\mathrm{c}} m_{\mathrm{p}} \ll e_{\mathrm{p}} m_{\mathrm{c}}$, it is possible for type 2 responses to intensify $\mathrm{IS}_{23}$ as the model transitions from a type 1 functional response.

\section{Numerical Solution for Protists}

In the main text, we used data sets for the body size dependence of population parameters to estimate the equilibrium abundances and calculate how the strength of a trophic cascade would change with the size of the top predator, which in this case is carnivorous protists. In the main text, we used a type 1 functional response. Here, we used a type 2 functional response and utilized the handling time data presented in the main text. Rather than reestimate all equilibrium abundances, here we simply estimate the equilibrium resource density in the three- and two-trophic-level models. There is an analytical solution for the resource abundance in a two-trophic-level model generated from the consumer's equation, $\hat{R}_{2}=m_{\mathrm{c}} /\left(e_{\mathrm{c}} a_{\mathrm{c}}-m_{\mathrm{c}} a_{\mathrm{c}} h_{\mathrm{c}}\right)$, where $m_{\mathrm{c}}$ is the mortality rate of the consumer, $e_{\mathrm{c}}$ is the conversion efficiency of the consumer, $a_{\mathrm{c}}$ is the area of capture of the consumer, and $h_{\mathrm{c}}$ is the consumer's handling time, as above. In contrast, the resource equilibrium in a three-trophic-level model must be evaluated numerically. We used the fzero command in Matlab to solve the resource equation for $\hat{R}_{3}$ across a range of top predator body sizes. Both resource abundances were estimated across the same set of top predator body sizes as in the main text, using the same allometric scaling relationships for the parameters shown in fig. 3 and table 1.

This analysis indicated that the trophic cascade follows the same general pattern of increasing with top predator body size as shown with the type 1 functional response (fig. A1). The strength of the trophic cascade for mean parameter estimates was slightly less than that with the type 1 functional response, which makes sense because the level of $\hat{R}_{2}$ is higher with a type 2 functional response (consumers can get a bit less of the resource when they have to handle the prey). At the smallest body sizes, there is a small region where at the mean parameter level the system is not viable because $e_{\mathrm{c}}<-m_{\mathrm{c}} h_{\mathrm{c}}$, which makes the value of $\hat{R}_{2}$ negative (see eq. [4]). Nonetheless, the overall pattern is similar and, in fact, statistically indistinguishable from the pattern predicted using a type 1 functional response. 


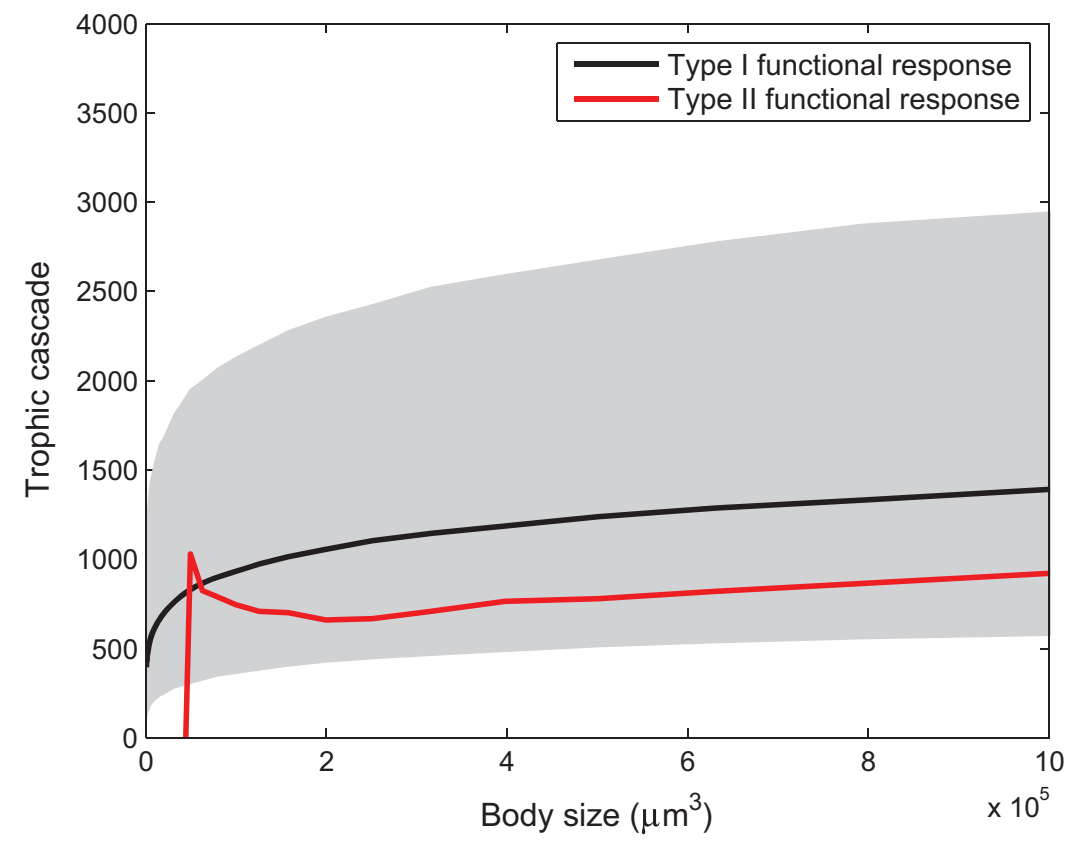

Figure A1: Body size dependence of a trophic cascade for protists using a type 1 functional response (black line $\pm 95 \%$ confidence intervals [gray area], from fig. 2). Drawn over that is the trophic cascade calculated using a type 2 functional response (red). Over most of the body size range, the strength of a trophic cascade shows the same basic body size dependence. At very small predator sizes, the system becomes unviable for traits predicted by allometric relationships, although there may still be many cases where systems may still be viable at small predator sizes. 